\title{
Microbial virulence, molecular epidemiology and pathogenic factors of fluoroquinolone-resistant Haemophilus influenzae infections in Guangzhou, China
}

CrossMark

Dingqiang Chen ${ }^{1 \dagger}$, Shuxian Wen ${ }^{2 \dagger}$, Donghua Feng ${ }^{2}$, Ruirui $\mathrm{Xu}^{3}$, Junyan Liu ${ }^{3,4^{*}}$, Brian M. Peters ${ }^{4}$, Danhong Su${ }^{2}$, Yongping Lin' ${ }^{2}$ Ling Yang ${ }^{2}$, Zhenbo $\mathrm{Xu}^{3,5,6^{*}}$ (D) and Mark E. Shirtliff ${ }^{6}$

\begin{abstract}
Background: Fluoroquinolone-resistant Haemophilus influenzae (FRHI) has been reported worldwide but remain unclear in China.

Methods: A total of 402 H. influenzae isolates collected from 2016 to 2017 were included. Antimicrobial susceptibility on 10 antibiotics was performed, and minimum inhibitory concentration of ciprofloxacin- and nalidixic acid-resistant strains were further determined by E-test strips, with risk factors also evaluated. Strains with resistance or reduced susceptibility to ciprofloxacin were subjected to sequencing of the quinolone resistance-determining regions (QRDR) and plasmid-mediated quinolone resistance genes by sequencing, with multi-locus sequence typing.

Results: $2.2 \%$ of $H$. influenzae strains were non-susceptible $(7 / 402,1.7 \%)$ or susceptible $(2 / 402,0.5 \%)$ to ciprofloxacin but NAL-resistant by E-test, and multidrug resistance was more common in fluoroquinolones non-susceptible $\mathrm{H}$. influenzae group $(p=0.000)$. Infection risk factors included invasive procedure $(p=0.011)$, catching cold/previous contact with someone who had a cold $(p=0.019)$, fluoroquinolones use during previous 3 months $(p=0.003)$. With none of mutations obtained in $g y r B$, parE and other plasmid-mediated quinolone resistance genes, 7 and 4 strains were found for Ser-84-Leu substitutions in gyrA and one amino acid substitution in the QRDR of gyrA linked with one amino acid substitution in the QRDR of parC, respectively. In addition, five sequence types (ST) were identified, with ST1719 firstly found.
\end{abstract}

Conclusions: For the first time, this study has reported the incidence, risk factors, molecular determinants on fluoroquinolones resistance and ST of FRHI strains in mainland China, representing the first evidence of mutation of gyrA and parC in China and the new ST1719 worldwide.

Keywords: Haemophilus influenzae, Fluoroquinolones, Quinolone resistance-determining regions, ST1719

\section{Background}

Haemophilus influenzae is a conditional pathogen that commonly colonizes in the nasopharynx of human beings. As a leading cause for community-acquired

\footnotetext{
*Correspondence: yaner0722@hotmail.com; zhenbo.xu@hotmail.com

${ }^{\dagger}$ Dingqiang Chen and Shuxian Wen contributed equally to this manuscript

${ }^{3}$ School of Food Science and Engineering, South China University of Technology, Guangzhou 510640, China

Full list of author information is available at the end of the article
}

pneumonia (CAP), H. influenza is also a major cause of acute otitis media (AOM) and bronchitis in young children [1], responsible for sever meningitis and septicemia responsible for 371,000 deaths per year globally [2].

Fluoroquinolones (FQs), a frequently used antibiotic for $H$. influenzae infections, was recommended for elderly patients or patients with underlying diseases according to the guidelines for CAP in Japan [3]. After the first report of FQs-resistant H. influenzae (FRHI) in 1993, this pathogen has spread worldwide and increased 
rapidly $[4,5]$. Though a number of previous studies on FRHI from Hong Kong and Taiwan were available, the incidence of FRHI was controversial [4, 6-10]. In Taiwan, resistance rate of fluoroquinolone has increased rapidly over recent years [4, 7], and levofloxacin-resistance $H$. influenzae has been found to spread in the nursing home residents $[7,11]$ and was related to regional predominance of certain STs [4, 12]. In Hong Kong, the carriage rate of $H$. influenzae was $28.5 \%$ in children aged 2-6 years and this was the first report about resistance to quinolones among $H$. influenzae isolates in children [6]. However, despite a regional cross-sectional study recently from Shanghai which reported an $8 \% \mathrm{H}$. influen$z a e$ carriage in healthy children (aged 12-18 months) and sensitivity to all tested antimicrobials except $12.2 \%$ ampicillin resistance to ampicillin (with none of FRHI found) [10], surveillance on FRHI still remains significantly limited in mainland China.

Although resistance to $\mathrm{FQs}$ in $H$. influenzae remains low, levofloxacin treatment failure in $H$. influenzae pneumonia has been reported [13]. A patient with $H$. influenzae pneumonia died after several days of systemic treatment with levofloxacin and several mutations in the quinolone resistance-determining regions (QRDR) of DNA gyrase and topoisomerase IV genes of the $H$. influenzae strain were detected [14]. Resistance to FQs is due mainly to chromosome-mediated mutations in the QRDRs of the genes encoding DNA gyrase and topoisomerase IV [15], which is including $\operatorname{gyr} A, \operatorname{gyr} B$, parC and parE. In general, resistance to $\mathrm{FQs}$ developed in a stepwise manner. Mutation in DNA gyrase gyrA, which was regarded as the first-step mutants, can even occur in $H$. influenzae that FQs MICs remains in the susceptible range according to the current breakpoints in CLSI [6]. Carmen et al. believe that FQ resistance is acquired gradually with increasing numbers of mutations [16]. Nalidixic acid can be used in tests to screen for the detection of decreased susceptibility to quinolones in $H$. influenzae $[6,17,18]$, it can find out isolates with QRDR mutant while quinolones MICs were still susceptible according to the breakpoint in CLSI.

In addition to the controversial FRHI incidence in Hong Kong and Taiwan and unavailability on FRHI study in mainland China [6, 7], antimicrobial resistance mechanism and sequence types (ST) of FRHI in mainland China remains unclear. In Guangzhou representative of Southern China, a number of longitudinal surveillances had been previously performed on various bacteria [1821], including antimicrobial resistance and molecular mechanisms. Conducted in the same location, this study aimed to investigate the molecular epidemiology and risk factors of FRHI infections.

\section{Methods}

\section{Species identification}

The study was carried out at The First Affiliated Hospital of Guangzhou Medical University, a comprehensive teaching hospital located in Guangzhou representative of Southern China and featured by the leading respiratory diseases study and treatment. $H$. influenzae isolates were recruited from patients during January 2016 to January 2017. The samples were grown on chocolate agars and incubated for $24 \mathrm{~h}$ at $37{ }^{\circ} \mathrm{C}$ in air with $5 \% \mathrm{CO}_{2}$. Suspicious colonies were removed from the surface of the plate for species identification, meanwhile, colonies (two to three) were homogenized in a reaction tube containing nanopure water $(100 \mu \mathrm{l})$ [22]. Species identification were performed by matrix-assisted laser desorption ionization time-of-flight mass spectrometry (MALDI-TOF MS, $\mathrm{AB}$ biomerieux), and confirmed by $16 \mathrm{~S}$ rRNA sequencing [23] (Primers were list in Table 1). DNA extracts were performed by boiling the bacterial suspensions [22]. The bacterial suspensions were heated to $100{ }^{\circ} \mathrm{C}$ in a boiling water bath for $10 \mathrm{~min}$ and centrifuged at $10,000 \times g$ for $10 \mathrm{~min}$, then DNA was used as template and amplified with the following polymerase chain reactions (PCR) procedure: denaturation at $95{ }^{\circ} \mathrm{C}$ for $5 \mathrm{~min}$, followed by 30 cycles of $95{ }^{\circ} \mathrm{C}$ for $30 \mathrm{~s}, 55^{\circ} \mathrm{C}$ for $30 \mathrm{~s}$, and $72{ }^{\circ} \mathrm{C}$ for $90 \mathrm{~s}$. The samples were then extended at $72{ }^{\circ} \mathrm{C}$ for a further $10 \mathrm{~min}$, hold to $4{ }^{\circ} \mathrm{C}$. The sequences of the PCR products were compared with known $16 \mathrm{~S}$ rRNA gene sequences in the GenBank (http://www.ncbi.nlm.nih. gov) as described previously [23, 24].

\section{Antimicrobial susceptibility}

Antimicrobial susceptibility of $H$. influenzae isolates to 10 antibiotics, including ampicillin (AMP), ampicillin-sulbactam (AMS), ciprofloxacin (CIP), levofloxacin (LEV), ceftriaxone (CTR), cefuroxime (ROXH), cefotaxime (TAX), meropenem (MEM), trimethoprim-sulfamethoxazole (SXT), azithromycin (AZT), was determined by Kirby-Bauer disk diffusion test (Oxoid, United Kingdom) on Haemophilus test medium (HTM) plates and incubated for $24 \mathrm{~h}$ at $37{ }^{\circ} \mathrm{C}$ in air with $5 \% \mathrm{CO}_{2}$. All the isolates were tested for the production of $\beta$-lactamase by nitrocephin paper disks.

Isolates that showed susceptibility to ciprofloxacin and had an inhibitory zone diameter of 21-28 $\mathrm{mm}$ were screened with nalidixic acid (NAL) disks $(30 \mu \mathrm{g})$. Minimum inhibitory concentrations (MICs) of ciprofloxacin and levofloxacin were detected by E-test method ( $A B$ bioMerieux) in isolates resistant to ciprofloxacin or NAL disks. Susceptibility results were interpreted according to CLSI standards 26th Edition except NAL screening. NAL screening was interpreted according to EUCAST criteria [16]. H. influenzae was considered decreased 
Table 1 Primers used in this study

\begin{tabular}{|c|c|c|c|}
\hline Genes & Primers & Sequences & References \\
\hline \multirow[t]{2}{*}{$16 S$ rRNA } & LPW55 & AGTTTGATCCTGGCTCAG & {$[19]$} \\
\hline & LPW56 & $\begin{array}{l}\text { AGGCCCGGGAACGTATTC } \\
\text { AC }\end{array}$ & \\
\hline \multirow[t]{2}{*}{ gyrA } & gyrA-F & CCGCCGCGTACTGTTCT & [21] \\
\hline & gyrA-R & CCATTTGCTAAAAGTGC & \\
\hline \multirow{2}{*}{ gyrB } & gyrB-F & GGAAAATCCTGCAGATGC & {$[21]$} \\
\hline & gyrB-R & AAGCAACGTACGGATGTG & \\
\hline \multirow[t]{2}{*}{ parc } & $\operatorname{parC}-\mathrm{F}$ & TGGTTTAAAACCCGTTCA & {$[21]$} \\
\hline & $\operatorname{parC}-\mathrm{R}$ & AGCAGGTAAATATTGTGG & \\
\hline \multirow[t]{2}{*}{ parE } & parE-F & GAACGCTTATCATCACGCCA & [21] \\
\hline & parE-R & $\begin{array}{l}\text { AGCATCCGCGAGAATACA } \\
\text { GA }\end{array}$ & \\
\hline \multirow[t]{2}{*}{ qnrA } & QnrA-F & $\begin{array}{l}\text { AGAGGATTTCTCACGCCA } \\
\text { GG }\end{array}$ & {$[22]$} \\
\hline & QnrA-R & $\begin{array}{l}\text { TGCCAGGCACAGATCTTG } \\
\text { AC }\end{array}$ & \\
\hline \multirow[t]{2}{*}{ anrB } & QnrB-F & $\begin{array}{l}\text { GGMATHGAAATTCGCCAC } \\
\text { TG }\end{array}$ & {$[22]$} \\
\hline & QnrB-R & TTTGCYGYYCGCCAGTCGAA & \\
\hline \multirow[t]{2}{*}{ anrs } & QnrS-F & $\begin{array}{l}\text { GCAAGTTCATTGAACAGG } \\
\text { GT }\end{array}$ & {$[22]$} \\
\hline & Qnrs-R & $\begin{array}{l}\text { TCTAAACCGTCGAGTTCG } \\
\text { GCG }\end{array}$ & \\
\hline \multirow[t]{2}{*}{$A a c\left(6^{\prime}\right)-1 b-c r$} & $a a c\left(6^{\prime}\right)-1 b-c r-F$ & $\begin{array}{l}\text { TTGCGATGCTCTATGAGT } \\
\text { GGCTA }\end{array}$ & {$[22]$} \\
\hline & $a a c\left(6^{\prime}\right)-1 b-c r-R$ & CTCGAATGCCTGGCGTGTTT & \\
\hline \multirow[t]{2}{*}{ qерA } & qepA-F & AACTGCTTGAGCCCGTAGAT & {$[22]$} \\
\hline & qepA-R & GTCTACGCCATGGACCTCAC & \\
\hline \multirow[t]{2}{*}{ oqxA } & oqxA-F & $\begin{array}{l}\text { AGTCCATACCAACCTCGT } \\
\text { CTCC }\end{array}$ & {$[22]$} \\
\hline & oqxA-R & GCGTGGCTTTGAACTCTGC & \\
\hline \multirow[t]{2}{*}{$o q \times B$} & $o q \times B-F$ & $\begin{array}{l}\text { CCACCCTTAACTGATCCC } \\
\text { TAA }\end{array}$ & {$[22]$} \\
\hline & $o q \times B-R$ & CGCCAGCTCATCCTTCAC & \\
\hline \multirow[t]{2}{*}{$a d k$} & $a d k-F$ & $\begin{array}{l}\text { GGTGCACCGGGTGCAGGT } \\
\text { AA }\end{array}$ & {$[23]$} \\
\hline & $a d k-R$ & CCTAAGATTTTATCTAACTC & \\
\hline \multirow[t]{2}{*}{$\operatorname{atp} G$} & $\operatorname{atpG-F}$ & $\begin{array}{l}\text { ATGGCAGGTGCAAAA } \\
\text { GAGAT }\end{array}$ & {$[23]$} \\
\hline & $\operatorname{atp} G-R$ & TTGTACAACAGGCTTTTGCG & \\
\hline \multirow[t]{2}{*}{$\operatorname{fr} d B$} & $\operatorname{frdB}-\mathrm{F}$ & CTTATCGTTGGTCTTGCCGT & {$[23]$} \\
\hline & $\operatorname{fr} d B-\mathrm{R}$ & TTGGCACTTTCCACTTTTCC & \\
\hline \multirow[t]{2}{*}{ fuck } & fuck-F & $\begin{array}{l}\text { ACCACTTTCGGCGTGGAT } \\
\text { GG }\end{array}$ & {$[23]$} \\
\hline & fuck-R & $\begin{array}{l}\text { AAGATTTCCCAGGTGCCA } \\
\text { GA }\end{array}$ & \\
\hline \multirow[t]{2}{*}{$m d h$} & $m d h-F$ & TCATTGTATGATATTGCCCC & {$[23]$} \\
\hline & $m d h-R$ & ACTTCTGTACCTGCATTTTG & \\
\hline \multirow[t]{2}{*}{ pgi } & pgi-F & GGTGAAAAAATCAATCGTAC & {$[23]$} \\
\hline & pgi-R & ATTGAAAGACCAATAGCTGA & \\
\hline \multirow[t]{2}{*}{ recA } & recA-F & $\begin{array}{l}\text { ATGGCAACTCAAGAAGAA } \\
\text { AA }\end{array}$ & [23] \\
\hline & $\operatorname{rec} A-\mathrm{R}$ & TTACCAAACATCACGCCTAT & \\
\hline
\end{tabular}

susceptibility to ciprofloxacin when MICs of ciprofloxacin were $\geq 0.125 \mu \mathrm{g} / \mathrm{ml}$. H. influenzae ATCC 29247 was used as a quality control strain for susceptibility testing.

\section{Sequencing of QRDRs and plasmid-mediated quinolone resistance genes}

Haemophilus influenzae showed decreased susceptibilities to ciprofloxacin were selected for molecular analysis. The isolates were detected for $\operatorname{gyr} A, \operatorname{gyr} B, \operatorname{par} C$ and parE mutations by using primers and methods described previously [25]. The amplification condition for QRDRs was as follows: denaturation at $95{ }^{\circ} \mathrm{C}$ for $5 \mathrm{~min}$ and 30 cycles of $95^{\circ} \mathrm{C}$ for $30 \mathrm{~s}$, annealing at $60^{\circ} \mathrm{C}$ for $1 \mathrm{~min}$, and polymerization at $72{ }^{\circ} \mathrm{C}$ for $90 \mathrm{~s}$; The samples were then extended at $72{ }^{\circ} \mathrm{C}$ for $7 \mathrm{~min}$ to fully extend the amplicons. The sequences of the PCR products were compared with known sequences $H$. influenzae Rd KW20 complete genome (L42023) in the GenBank. Plasmid mediated quinolone resistance (PMQR) genes qnrA, qnrB, qnrS, $a a c\left(6^{\prime}\right)-I b-c r, q e p A, o q x A$, and $o q x B$ were amplified and sequenced as described previously [26] (Table 1 ).

\section{Nucleotide accession numbers}

The sequences of 11 strains with gyrA or parC mutations have been annotated and submitted to the GenBank under accession number MG694554, MG694555, MG694556, MG694557, MG694558, MG694559, MG694560, MG694561, MG694562, MG694563 and MG694564.

\section{Multilocus sequence typing (MLST)}

Seven housekeeping genes fragments including $a d k$, atp $G$, frdB, fuck, $m d h$, pgi, recA were amplified according to the primers in Table 1. PCR products were obtained with the following PCR amplification procedure, initial denaturation at $95{ }^{\circ} \mathrm{C}$ for $4 \mathrm{~min}$, followed by 30 cycles of $95{ }^{\circ} \mathrm{C}$ for $30 \mathrm{~s}, 55^{\circ} \mathrm{C}$ for $30 \mathrm{~s}$, and $72{ }^{\circ} \mathrm{C}$ for $60 \mathrm{~s}$. The samples were then maintained at $72^{\circ} \mathrm{C}$ for $10 \mathrm{~min}$, cooled to $4{ }^{\circ} \mathrm{C}$. Allele numbers and STs were assigned by applying the $H$. influenzae MLST website (http://haemophilu s.mlst.net/) [27].

\section{Statistical analysis}

Data were analyzed with the Statistical Package for Social Sciences for Windows (SPSSWIN, Chicago, IL, USA, version 19.0). Continuous data, shown as mean $\pm S D$ or medians, were compared by Student's t-test or the Mann-Whitney $U$-test. Categorical variables, summarized as numbers and percentages, were compared by Chi square-test or Fisher's exact test. Two sided $p$-values less than 0.05 were considered statistically significant. 


\section{Results}

\section{Bacterial isolates}

As clinical data of the studied 402 nonduplicate $H$. influenzae strains was concerned, male patients accounted for 63.7\% ( $\mathrm{n}=256)$, and 130 (32.3\%), $110(27.4 \%)$ and 162 (40.3\%) patients were $\geq 60$ (mean: $79.41 \pm 8.77$ ), 15-59 (mean: $50.50 \pm 15.56$ ) and $<15$ (mean: $50.50 \pm 15.56$ ) years old, respectively. $H$. influenzae strains were originated from different anatomical sites: sputum $(\mathrm{n}=258$, $64.1 \%)$, throat swab $(n=64,15.9 \%)$, bronchoscope sputum $(\mathrm{n}=48,11.9 \%)$, bronchoalveolar lavage fluid $(\mathrm{n}=19$, $4.7 \%)$, secretion $(\mathrm{n}=13,3.2 \%) . H$. influenzae isolates were obtained from patients admitted to the respiratory department $(\mathrm{n}=141,35.0 \%)$, pediatrics department $(\mathrm{n}=163,40.5 \%)$, otolaryngology department $(\mathrm{n}=12$, $3.0 \%)$, thoracic surgery $(n=10,2.5 \%)$, nephrology department $(n=8,2 \%)$, allergy department $(n=7,1.7 \%)$, radiotherapy department $(n=7,1.7 \%)$, others $(n=54,13.4 \%)$.

\section{Sensitivity of $\boldsymbol{H}$. influenzae isolates}

Of the $402 \mathrm{H}$. influenzae isolates, one hundred and sixtynine $(42.0 \%)$ possessed $\beta$-lactamases and were resistant to ampicillin. Eighty-nine isolates were $\beta$-lactamase negative but resistant to ampicillin which were defined as BLNAR ( $\beta$-lactamase-negative ampicillin-resistant). Forty isolates were totally susceptible to ten antimicrobials according to Kirby-Bauer disk diffusion test, while $259(64.4 \%)$ strains were resistant to sulfamethoxazole, followed with ampicillin $(n=247,61.4 \%)$, cefuroxime ( $\mathrm{n}=114,28.4 \%)$, azithromycin $(\mathrm{n}=70,17.4 \%)$, cefotaxime $(n=45,11.2 \%)$, ceftriaxone $(n=37,9.2 \%)$, meropenem $(n=21,5.2 \%)$. Multidrug resistant strains accounted for 61 (15.2\%).

\section{Ciprofloxacin-nonsusceptible $\boldsymbol{H}$. influenzae isolates}

Eleven (2.7\%) ciprofloxacin-resistant $H$. influenzae were detected by the Kirby-Bauer disk diffusion test and seven (1.7\%) with reduced susceptibilities or resistant to ciprofloxacin (MICs were range from 0.5 to $12 \mu \mathrm{g} / \mathrm{ml}$ ) were detected by the E-test method. The MICs of levofloxacin ranged from 0.5 to $3 \mu \mathrm{g} / \mathrm{ml}$. Among $402 \mathrm{H}$. influen$z a e$, fifteen $(3.7 \%)$ isolates with ciprofloxacin inhibitory zone diameters of 21-28 mm were screened by the NAL disks, 2 of which were NAL-resistant with inhibitory zone diameters less than $23 \mathrm{~mm}$. The ciprofloxacin MICs of NAL-resistant strains were $0.38 \mu \mathrm{g} / \mathrm{ml}$ and $0.5 \mu \mathrm{g} / \mathrm{ml}$, respectively. The susceptibility of ciprofloxacin-nonsusceptible $H$. influenzae isolates were shown in Table 3.

\section{Infection risks analysis}

The comparison of ciprofloxacin nonsusceptible to susceptible group according to 17 clinical variables is listed in Table 2, which is including sex, underlying diseases, causes, and medical history during previous 3 months. Among the causes, receiving invasive procedure like sputum suction and bronchoscopy $(p=0.011)$, catching cold or previous contact with someone who had a cold $(p=0.019)$ and fluoroquinolones use during previous 3 months $(p=0.003)$ can increased the risks of ciprofloxacin-nonsusceptible $H$. influenzae infection (Table 2). Moreover, multidrug resistant strains were more common to the ciprofloxacin-nonsusceptible group $(p=0.000)$.

\section{Detection of amino acid substitutions in the QRDRs and plasmid-mediated quinolone resistance genes}

Seven isolates harbored the amino acid substitution Ser84-Leu in gyrA while four isolates harbored the amino acid substitution Ser-84-ILe in parC. Four isolates had both two amino acid substitutions in QRDRs. Three of the isolates with mutations were obtained from young children with 1-3 years of age. The MICs of ciprofloxacin to strains with one or two mutations were of $0.38-1.5 \mu \mathrm{g} /$ $\mathrm{ml}$, while one strain obtained from an outpatient with MIC of $12 \mu \mathrm{g} / \mathrm{ml}$ did not harbored alterations in QRDRs. No amino acid substitutions were found in $\operatorname{gyr} B$ and $p a r E$ genes and no plasmid-mediated quinolone resistance genes were found in our investigation (Table 3).

\section{Multilocus sequence typing (MLST)}

Nine ciprofloxacin-nonsusceptible isolates were resolved into 5 different sequence types (ST), which displayed high genetic variability. Four STs were previously described in the MLST database and one (ST1791) was a new ST that had not been reported previously. ST408 was the most common ST in our study. However, according to the epidemiological data such as the isolation date and departments, no clonal spread of FRHI was found in our study.

\section{Discussion}

This study has provided data of ciprofloxacin-resistant $H$. influenzae incidence in Guangzhou, China. The incidence in our study was closer to the report from Hong Kong [6], which stayed at a very low resistant rate, in contrary to Taiwan's [4, 8]. According to our investigation, the use of fluoroquinolone was not so common in adults, which may explain the low incidence of ciprofloxacinresistant $H$. influenzae in Guangzhou. Although most of the $H$. influenzae were community-acquired, invasive procedure like sputum suction and bronchoscopy may also increase the infection risks of ciprofloxacin-resistant $H$. influenzae which indicated that nosocomial environment colonization is an important route of ciprofloxacinresistant $H$. influenzae infection. In addition, medication history of fluoroquinolone during previous 3 months is closely related to ciprofloxacin-resistant $H$. influenzae 
Table 2 Clinical characteristics and infection risk factors of Haemophilus influenzae with resistance or reduced susceptibility to ciprofloxacin/levofloxacin

\begin{tabular}{|c|c|c|c|c|}
\hline \multirow[t]{2}{*}{ Variables $^{\mathrm{a}}$} & \multirow[t]{2}{*}{$\mathrm{N}$} & \multicolumn{2}{|c|}{ Ciprofloxacinc } & \multirow[t]{2}{*}{$p^{d}$} \\
\hline & & Susceptible & Decreased susceptibility & \\
\hline \multicolumn{5}{|l|}{ Age (years) } \\
\hline $0-14$ & $162(40.3 \%)$ & & & \\
\hline $5-59$ & $110(27.4 \%)$ & & & \\
\hline$\geq 60$ & $130(32.3 \%)$ & & & \\
\hline \multicolumn{5}{|l|}{ Sex } \\
\hline Male & $256(63.7 \%)$ & $250(63.6 \%)$ & $6(66.7 \%)$ & 1.000 \\
\hline Female & $146(36.3 \%)$ & $143(36.3 \%)$ & $3(33.3 \%)$ & 1.000 \\
\hline \multicolumn{5}{|l|}{ Prevalent seasons (months) } \\
\hline $3-5$ & $138(34.3 \%)$ & & & \\
\hline $6-8$ & $67(16.7 \%)$ & & & \\
\hline $9-11$ & $54(13.4 \%)$ & & & \\
\hline $12-2$ & $143(35.6 \%)$ & & & \\
\hline \multicolumn{5}{|l|}{ Underlying diseases } \\
\hline Chronic obstructive pulmonary disease & $60(14.9 \%)$ & $58(14.7 \%)$ & $2(22.2 \%)$ & 0.626 \\
\hline Hypertension & $70(17.4 \%)$ & $68(17.3 \%)$ & $2(22.2 \%)$ & 1.000 \\
\hline Diabetes & $28(7.0 \%)$ & $27(6.9 \%)$ & $1(11.1 \%)$ & 1.000 \\
\hline Chronic kidney disease & $33(8.2 \%)$ & $33(8.4 \%)$ & $0(0 \%)$ & $1.000^{\mathrm{b}}$ \\
\hline Cancers & $46(11.4 \%)$ & $45(11.5 \%)$ & $1(11.1 \%)$ & 1.000 \\
\hline Interstitial lung Disease & $14(3.5 \%)$ & $13(3.3 \%)$ & $1(11.1 \%)$ & 0.732 \\
\hline Bronchiectasia & $27(6.7 \%)$ & & & \\
\hline \multicolumn{5}{|l|}{ Causes } \\
\hline Catch cold or be tired & $30(7.5 \%)$ & $27(6.9 \%)$ & $3(33.3 \%)$ & 0.019 \\
\hline Invasive procedure & $27(6.7 \%)$ & $24(6.1 \%)$ & $3(33.3 \%)$ & 0.011 \\
\hline History of smoking & $40(9.9 \%)$ & $39(9.9 \%)$ & $1(11.1 \%)$ & 1.000 \\
\hline \multicolumn{5}{|l|}{ Channel of infection } \\
\hline Community-acquired & $372(92.5 \%)$ & & & \\
\hline Hospital-acquired & $30(7.5 \%)$ & & & \\
\hline Hospitalization during previous 3 months & $133(33.1 \%)$ & $128(32.6 \%)$ & $5(55.6 \%)$ & 0.147 \\
\hline Fluoroquinolones use during previous 3 months & $5(1.2 \%)$ & $7(1.8 \%)$ & $2(22.2 \%)$ & 0.003 \\
\hline \multicolumn{5}{|l|}{ Antimicrobial use during previous 3 months } \\
\hline Cephalosporins & & $144(36.6 \%)$ & $4(44.4 \%)$ & 0.896 \\
\hline Azithromycin & & $70(17.8 \%)$ & $0(0 \%)$ & $0.370^{b}$ \\
\hline Piperacillin & & $13(3.3 \%)$ & $1(11.1 \%)$ & 0.732 \\
\hline Multidrug resistant strains & & $55(14.0 \%)$ & $8(88.9 \%)$ & 0.000 \\
\hline
\end{tabular}

a Data are presented as mean \pm SD or number of patients (\%)

b Fisher's exact test

c Numbers of susceptible and decreased susceptibility are 393 and 9, respectively

d Statistically significant data are in italics

infection. Patients who are reinfected with $H$. influenzae should be monitored of fluoroquinolone susceptibility for the isolates may have obtained resistance under antibiotic selective pressure. It is known that fluoroquinolone is not approved for children in most regions of the world now, and children infected with ciprofloxacin-resistant $H$. influenzae in our study were all community-acquired, so we inferred that the acquisition of such $H$. influenzae in children may be attributed to disseminating from community environment and consuming products from food animal previously fed antimicrobial agents of fluoroquinolone. In addition, $H$. influenzae carriage in children should rise our concern in preventing the spread of resistant $H$. influenzae, especially in public areas like daycares [6]. 


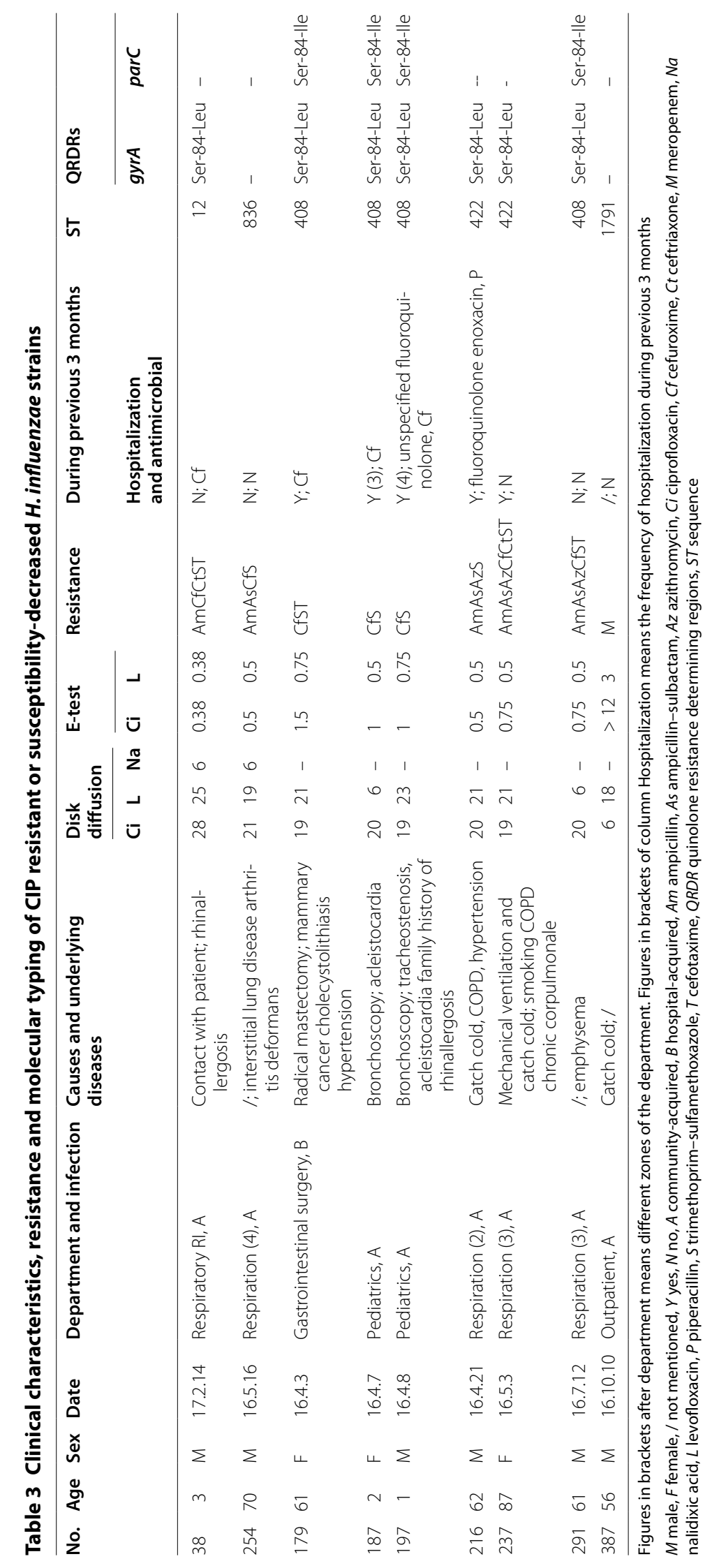


Although ciprofloxacin-resistant $H$. influenzae remains rare in Guangzhou, nine ciprofloxacin-nonsusceptible. $H$. influenzae isolates were detected in our study $(2.2 \%$, including two NAL-resistant strains), which may develop into resistance in a stepwise manner according to the molecular mechanism in QRDRs. Seven of the isolates had developed amino acid substitution in QRDRs, with ciprofloxacin MICs of 0.38 to $1.5 \mu \mathrm{g} / \mathrm{ml}$. H. influenzae in our investigation with ciprofloxacin MICs of 0.38$0.75 \mu \mathrm{g} / \mathrm{ml}$ had one amino acid alteration in $g y r A$, and isolates with ciprofloxacin MICs of $0.75-1.5 \mu \mathrm{g} / \mathrm{ml}$ had two amino acid alteration in $g y r A$ and parC. According to the previous report, isolates with ciprofloxacin MICs of $0.12-0.5 \mu \mathrm{g} / \mathrm{ml}$ had at least one amino acid alteration in a position of the fluoroquinolone targets involved in resistance. It was revealed that the degree of fluoroquinoloneresistance is related to the number of the mutations in QRDRs [16]. Strains presenting one or two mutations in gyrA and parC have low-level resistance to fluoroquinolone, while those with three or more mutations show high-level resistance [28]. In general, our finding was consistent with the report [16]. However, there were two isolates with ciprofloxacin MICs of $0.5 \mu \mathrm{g} / \mathrm{ml}$ and $12 \mu \mathrm{g} /$ $\mathrm{ml}$ respectively didn't harboring any mutations in QRDRs and plasmid-mediated quinolone resistance genes. We inferred that some other mechanisms may participated in fluoroquinolone-resistance of $H$. influenzae.

Amino acid changes in gyrA residue (Ser84) and/or parC residue (Ser84) were detected in our study while no substitutions occurred in $\operatorname{gyr} B$ and parE. Ser84Leu in $g y r A$ and Ser-84-Ile in parC were globally reported according to the current literatures, including Spain, Japan, Taiwan $[4,14,16,25,29]$. The similar mutation patterns in QRDRs around different areas indicated that Ser-84-Leu in gyrA and Ser-84-Ile in parC were one of the most common mutations in QRDRs. Comparing with the mutation profiles in QRDRs from Guangzhou and Taiwan, the latter one had more diverse mutation profiles than Guangzhou [4]. In Taiwan, emergence of fluoroquinolone-resistant isolates may be the results of several clones spreading in the same region. However, according to our investigation, no clone transmitted hospital infection was found which indicated that fluoroquinolone-nonsusceptible $H$. influenzae may occur sporadically in Guangzhou presently. In addition, our investigation reported a relative high genetic diversity among fluoroquinolone-resistant $H$. influenzae isolates. None of the STs published in Taiwan were found in our study, but ST422 and ST408 involved in our study were previously reported in Sichuan, China, where also discovered a series of diverse and novel sequence types as Taiwan [4, 30]. The predominant epidemiological STs in China like ST486 and 480 were not found in our study.
The heterogeneous characteristics of the $H$. influenzae strains may be the reason that no outbreak or epidemics occurred in Guangzhou. Moreover, high heterogeneity of $H$. influenzae strains by MLST analysis revealed that the $H$. influenzae strains were regionally varied and evolved independently. One strain was confirmed as novel sequence type (ST1719) in our study, which showed high-level resistance of ciprofloxacin while no mutations in QRDRs was found.

There were two NAL-resistant strains harboring amino acid substitution in QRDRs in our study and both of them were ciprofloxacin-susceptible according to CLSI criteria. It has been argued that, the application of current CLSI criteria may underrecognize the low-level ciprofloxacin-resistant $H$. influenzae with first step mutations in QRDRs $[6,16,17]$. As a screening test for determination of the susceptibilities of gram-negative bacteria to fluoroquinolone, NAL has drawn special attention $[6,16]$, it can separate strains with reduced quinolone susceptibilities as a result of the acquisition of mutations in the QRDRs. However, NAL screening was not applied routinely in determination of the susceptibilities of $H$. influenzae in clinics which may underrecognize a proportion of strains with decreased fluoroquinolone susceptibilities. In addition, no acceptable criteria for NAL screening was established by CLSI in the current. In order to detect fluoroquinolone non-susceptible strains with first step mutation in QRDRs, screening with NAL as an indicator of resistance was proposed by the EUCAST guidelines. First failure case of oral levofloxacin treatment of community-acquired pneumonia caused by $H$. influenzae had been reported in 2003 [14]. After that, reports on fluoroquinolone treatment failure were occurred globally. An reports from New York declared that persistent stimulation of low concentration fluoroquinolone in fluoroquinolone-susceptible $H$. influenzae may increase the fluoroquinolone resistance of $H$. influenzae isolates which offered experiment evidence for the declaration of avoiding fluoroquinolone treatment in $H$. influenzae close to breakpoints. And they pointed out that the key to preventing fluoroquinolone resistance in $H$. influenzae may be to block enrichment of the first gyrA mutation by strictly avoiding low dose fluoroquinolones use [28]. In addition, medication history of fluoroquinolone is related to fluoroquinolone non-susceptible $H$. influenzae infection according to our investigation, so we inferred that a proportion of strains harboring the first step mutation in QRDRs might obtain fluoroquinolone resistance under fluoroquinolone selection pressure. High-level resistance might be induced by repeated treatment with fluoroquinolone in patients with chronic lung diseases like COPD. Treatment failure reminded us that we should pay attention to those $H$. influenzae with resistance or decreased 
susceptibilities to fluoroquinolone, and should facilitate the laboratory detection of strains with decreased susceptibilities to fluoroquinolone.

In conclusion, as the first study of FRHI in mainland China, reduced susceptibilities to fluoroquinolones had been obtained, despite low incidence of FRHI isolates. Invasive procedure and medication history of fluoroquinolone during previous 3 months can increase the infection risks of FRHI. High genetic diversity in FRHI strains has strongly suggested their sporadic emergence and spread in Guangzhou of Southern China, and amino acid substitutions in QRDRs of strains with decreased susceptibilities to fluoroquinolone have also presented evidence on the initial evolution of fluoroquinolone resistance by mutation in $g y r A$, indicating the potential treatment failure or increase of fluoroquinolone resistance under continuous fluoroquinolone therapy. In addition, this study has for the first time reported the mutation of $g y r A$ and parC in mainland China and the new ST1719 worldwide, which may further raise clinical concerns regarding the therapeutic efficacy of fluoroquinolone treatment and aid in further investigation on FRHI.

\begin{abstract}
Abbreviations
FRHI: fluoroquinolone-resistant Haemophilus influenzae; QRDR: quinolone resistance-determining regions; CAP: community-acquired pneumonia; $\mathrm{AOM}$ : acute otitis media; FQs: fluoroquinolones; ST: sequence types; AMP: ampicillin; AMS: ampicillin-sulbactam; CIP: ciprofloxacin; LEV: levofloxacin; CTR: ceftriaxone; ROXH: cefuroxime; TAX: cefotaxime; MEM: meropenem; SXT: trimethoprim-sulfamethoxazole; AZT: azithromycin; HTM: Haemophilus test medium; NAL: nalidixic acid; MICs: minimum inhibitory concentrations; PMQR: plasmid mediated quinolone resistance.
\end{abstract}

\section{Authors' contributions}

DC and LY designed the study, SW and DF carried out the experiments, including species identification, antimicrobial susceptibility, YL and DS conducted the experiment of the sequencing of QRDRs and analyzed the plasmid-mediated quinolone resistance genes, etc. ZX, $L$ and RX performed the statistical analysis, and wrote the manuscript. All authors read and approved the final manuscript.

\begin{abstract}
Author details
1 Department of Laboratory Medicine, Zhujiang Hospital, Southern Medical University, Guangzhou 510280, China. ${ }^{2}$ Department of Laboratory Medicine, The First Affiliated Hospital of Guangzhou Medical University, Guangzhou Medical University, Guangzhou 510120, China. ${ }^{3}$ School of Food Science and Engineering, South China University of Technology, Guangzhou 510640, China. ${ }^{4}$ Department of Clinical Pharmacy, College of Pharmacy, University of Tennessee Health Science Center, Memphis, TN 38163, USA. ${ }^{5}$ Guangdong Province Key Laboratory for Green Processing of Natural Products and Product Safety, Guangzhou 510640, China. ${ }^{6}$ Department of Microbial Pathogenesis, School of Dentistry, University of Maryland, Baltimore, MD 21201, USA.
\end{abstract}

\section{Acknowledgements}

This work was supported by the Natural Science Foundation of Guangdong Province (2018A030310170 and 2018A030313279), the National Key Research and Development Program of China (2016YFD04012021), Guangdong Special Support Program (2016TQ03N682), Pearl River S\&T Nova Program of Guangzhou (201710010061), Science and Technology Planning Project of Guangdong Province (2017A050501007), National Outstanding Doctoral Dissertation Funding (201459), and the Fundamental Research Funds for the Central Universities (2017ZD092).

\section{Competing interests}

The authors declare that they have no competing interests.

Availability of data

Please contact author for data requests.

\section{Consent for publication}

Not applicable.

Ethics approval and consent to participate

Not applicable.

\section{Publisher's Note}

Springer Nature remains neutral with regard to jurisdictional claims in published maps and institutional affiliations.

Received: 17 March 2018 Accepted: 9 October 2018

Published online: 23 November 2018

\section{References}

1. Garcia-Cobos S, Moscoso M, Pumarola F, Arroyo M, Lara N, Perez-vazquez $M$, et al. Frequent carriage of resistance mechanisms to beta-lactams and biofilm formation in Haemophilus influenzae causing treatment failure and recurrent otitis media in young children. J Antimicrob Chemother. 2014;69:2394-9.

2. Watt JP, Wolfson $L$, O'Brien $K L$, Henkle E, Deloria-Knoll M, Natelie MM, et al. Burden of disease caused by Haemophilus influenzae type $b$ in children younger than 5 years: global estimates. Lancet. 2009;374:903-11.

3. Committee for The Japanese Respiratory Society guidelines for the management of respiratory infections. Guidelines for the management of community acquired pneumonia in adults, revised edition. Respirology. 2006;11:S79-133.

4. Kuo S, Chen P, Shiau YR, Wang H, Lai J, Huang IW, et al. Levofloxacinresistant Haemophilus influenzae, Taiwan, 2004-2010. Emerg Infect Dis. 2014;20:1386-90

5. Li X, Mariano N, Rahal JJ, Urban CM, Drlica K. Quinolone-resistant Haemophilus influenzae in a long-term-care facility: nucleotide sequence characterization of alterations in the genes encoding DNA gyrase and DNA topoisomerase IV. Antimicrob Agents Chemother. 2004;48:3570-2.

6. Pak LH, Chow KH, Mak GC, Tsang KW, Lau YL, Ho AY, et al. Decreased levofloxacin susceptibility in Haemophilus influenzae in children, Hong Kong. Emerg Infect Dis. 2004;10:1960.

7. Chang C, Lauderdale TL, Lee HC, Lee NY, Wu C, Chen P, et al. Colonisation of fluoroquinolone-resistant Haemophilus influenzae among nursing home residents in southern Taiwan. J Hosp Infect. 2010;75:304-8.

8. Hu F, Zhu D, Wang F, Morrissey I, Wang J, Torumkuney D. Results from the survey of antibiotic resistance (SOAR) 2009-11 and 2013-14 in China. J Antimicrob Chemother. 2016;71:33-43.

9. Miao J, Liang Y, Chen L, Wang W, Wang J, Li B, Li L, Chen D, Xu Z. Formation and development of biofilm: with focus on food safety. J Food Saf. 2017;37(4):e12358.

10. Hu J, Sun X, Huang Z, Wagner A, Carlson B, Yang J, et al. Streptococcus pneumoniae and Haemophilus influenzae type $b$ carriage in Chinese children aged 12-18 months in Shanghai, China: a cross-sectional study. BMC Infect Dis. 2016;16:149.

11. Lin J, Chen X, Chen C, Hu J, Zhou C, Cai X, Wang W, Zheng C, Zhang P, Cheng J, Guo Z, Liu H. Durably antibacterial and bacterially antiadhesive cotton fabrics coated by cationic fluorinated polymers. ACS Appl Mater Interfaces. 2018;10(7):6124-36.

12. Liu J, Chen D, Peters BM, Li L, Li B, Xu Z, Shirliff ME. Staphylococcal chromosomal cassettes mec (SCCmec): a mobile genetic element in methicillin-resistant Staphylococcus aureus. Microb Pathog. 2016;101:56-67.

13. Vila J, Ruiz J, Sanchez F, Navarro F, Mirelis B, de Anta MT, et al. Increase in quinolone resistance in a Haemophilus influenzae strain isolated from a patient with recurrent respiratory infections treated with of ofloxacin. Antimicrob Agents Chemother. 1999;43:161-2. 
14. Bastida T, Perez-Vazquez M, Campos J, Cortes-Lletget MC, Roman F, Tubau F, et al. Levofloxacin treatment failure in Haemophilus influenzae pneumonia. Emerg Infect Dis. 2003;9:475-1478.

15. Georgiou M, Munoz R, Roman F, Canton R, Gomez-Lus R, Campos J, et al. Ciprofloxacin-resistant Haemophilus influenzae strains possess mutations in analogous positions of GyrA and ParC. Antimicrob Agents Chemother. 1996;40:1741-4

16. Puig C, Tirado-Velez JM, Calatayud L, Tubau F, Garmendia J, Ardanuy $C$, et al. Molecular characterization of fluoroquinolone resistance in nontypeable Haemophilus influenzae clinical isolates. Antimicrob Agents Chemother. 2015;59:461-6.

17. Perez-Vazquez M, Roman F, Aracil B, Canton R, Campos J. Laboratory detection of Haemophilus influenzae with decreased susceptibility to nalidixic acid, ciprofloxacin, levofloxacin, and moxifloxacin due to gyr $A$ and parC mutations. J Clin Microbiol. 2004;42:1185-91.

18. Xu Z, Xie J, Peters BM, Li B, Li L, Yu G, Shirtliff ME. Longitudinal surveillance on antibiogram of important Gram-positive pathogens in Southern China, 2001 to 2015. Microb Pathog. 2017:103:80-6.

19. Xie J, Yang L, Peters BM, Chen L, Chen D, Li B, et al. A 16-year retrospective surveillance report on the pathogenic features and antimicrobial susceptibility of Pseudomonas aeruginosa isolates from FAHJU in Guangzhou representative of Southern China. Microb Pathog. 2017;110:37-41.

20. Xie J, Peters BM, Li B, Li L, Yu G, Xu Z, et al. Clinical features and antimicrobial resistance profiles of important Enterobacteriaceae pathogens in Guangzhou representative of Southern China, 2001-2015. Microb Pathog. 2017; 107:206-11.

21. Liu J, Deng Y, Peters BM, Li L, Li B, Chen L, et al. Transcriptomic analysis on the formation of the viable putative non-culturable state of beer-spoilage Lactobacillus acetotolerans. Sci Rep. 2016;6:1-11.

22. McBain AJ, Bartolo RG, Catrenich CE, Charbonneau D, Ledder RG, Gilbert P. Effects of triclosan-containing rinse on the dynamics and antimicrobial susceptibility of in vitro plaque ecosystems. Antimicrob Agents Chemother. 2003;47:3531-8.

23. Lau SKP, Woo PCY, Mok MY, Teng JLL, Tam VKP, Chan KKH, et al. Characterization of Haemophilus segnis, an important cause of bacteremia, by $16 \mathrm{~S}$ rRNA gene sequencing. J Clin Microbiol. 2004;42:877-80.

24. Thompson JD, Higgins DG, Gibson TJ, Clustal W. Improving the sensitivity of progressive multiple sequence alignment through sequence weighting, position-specific gap penalties and weight matrix choice. Nucleic Acids Res. 1994:22:4673-80

25. Shoji H, Shirakura T, Fukuchi K, Takuma T, Hanaki H, Tanaka K, et al. A molecular analysis of quinolone-resistant Haemophilus influenzae: validation of the mutations in quinolone resistance-determining regions. J Infect Chemother. 2014;20:250-5.

26. Al-Agamy MH, Aljallal A, Radwan HH, Shibl AM. Characterization of carbapenemases, ESBLs, and plasmid-mediated quinolone determinants in carbapenem-insensitive Escherichia coli and Klebsiella pneumoniae in Riyadh hospitals. J Infect Public Health. 2017;731:1-5.

27. Meats E, Feil EJ, Stringer S, Cody AJ, Goldstein R, Kroll JS, et al. Characterization of encapsulated and noncapsulated Haemophilus influenzae and determination of phylogenetic relationships by multilocus sequence typing. J Clin Microbiol. 2003;41:1623-36.

28. Li X, Mariano N, Rahal JJ, Urban CM, Drlica K. Quinolone-resistant Haemophilus influenzae: determination of mutant selection window for ciprofloxacin, garenoxacin, levofloxacin, and moxifloxacin. Antimicrob Agents Chemother. 2004;48:4460-2

29. Yokot S, Ohkoshi Y, Sato K, Fujii N. Emergence of fluoroquinoloneresistant Haemophilus influenzae strains among elderly patients but not among children. J Clin Microbiol. 2008;46:361-5.

30. Tian G, Zhang L, Li M, Wang X, Zheng Y, Li X, et al. Genotypic characteristics of Haemophilus influenzae isolates from pediatric pneumonia patients in Chengdu city, Sichuan, China. J Microbiol. 2009;47:494-7.
Ready to submit your research? Choose BMC and benefit from:

- fast, convenient online submission

- thorough peer review by experienced researchers in your field

- rapid publication on acceptance

- support for research data, including large and complex data types

- gold Open Access which fosters wider collaboration and increased citations

- maximum visibility for your research: over $100 \mathrm{M}$ website views per year

At BMC, research is always in progress.

Learn more biomedcentral.com/submissions 